\title{
Time-Resolved Dynamics of Shallow Acceptor Transitions in Silicon
}

\author{
N. Q. Vinh, ${ }^{1,2}$ B. Redlich, ${ }^{1,3}$ A. F. G. van der Meer, ${ }^{1,3}$ C. R. Pidgeon, ${ }^{4}$ \\ P. T. Greenland, ${ }^{5}$ S. A. Lynch, ${ }^{5}$ G. Aeppli, ${ }^{5}$ and B. N. Murdin ${ }^{6}$ \\ ${ }^{1}$ FOM Institute for Plasma Physics "Rijnhuizen", P. O. Box 1207, 3430 BE Nieuwegein, The Netherlands \\ ${ }^{2}$ Department of Physics, Virginia Tech, Blacksburg, Virginia 24061, USA \\ ${ }^{3}$ Radboud University, Institute for Molecules and Materials, FELIX Facility, Toernooiveld 7, 6525 ED Nijmegen, The Netherlands \\ ${ }^{4}$ Heriot-Watt University, Department of Physics, Riccarton, Edinburgh, EH14 4AS, United Kingdom \\ ${ }^{5}$ London Centre for Nanotechnology, University College London, London WC1H OAH, United Kingdom \\ ${ }^{6}$ Advanced Technology Institute and SEPNet, University of Surrey, Guildford, GU2 7XH, United Kingdom \\ (Received 7 September 2012; revised manuscript received 17 December 2012; published 14 March 2013)
}

Shallow group-V donors in silicon may be thought of as hydrogenlike, and shallow acceptors are similarly described by effective-mass theory with similar energy scales, which implies that donor and acceptor excitations should be just as long-lived. Yet, spectral widths of acceptors are considerably wider. We have measured the orbital dynamics of acceptors in silicon using time-domain spectroscopy with a free-electron laser. Both the population and coherence lifetimes for acceptors in natural silicon are substantially longer-e.g. approximately $60 \mathrm{ps}$ for boron-than implied by the spectral linewidths; our experiments also establish the recombination time for ionized acceptors to be, at approximately $500 \mathrm{ps}$, nearly an order of magnitude longer. We show that there are no extra sources of decoherence introduced by the host crystal, other than the population relaxation. In this sense, the crystal acts as an atom trap, and, by introducing quantum coherent control of acceptors to that previously established for donors, we open the way to optically controllable nanoscale p-n devices.

DOI: 10.1103/PhysRevX.3.011019

\section{INTRODUCTION}

Impurities in solids are receiving a resurgence of interest because of their close analogy with free atoms. Where free atoms have found application in quantum information [1,2], semiconductor impurities are being developed for atomic-scale spintronics and orbitronics [3-8], and alkalivapor-laser concepts [9] are used in semiconductor gain media [10,11]. The electrons bound to group- $\mathrm{V}$ donor atoms in Si occupy hydrogenlike wave functions, and many fundamental parameters such as their energy and Bohr radius can be predicted by simple rescaling of the electron mass and dielectric constant from their free-space values $[6,12]$.

Both donors and acceptors are available, and the underlying hydrogenic picture of the $1 / r$ potential applies to both but is modified in different ways in each case. The symmetry of the acceptor wave functions (shown in Fig. 1 and compared with those of shallow donors) means that the states are easily and controllably split by strain, electric fields, or quantum well confinement, which is useful for the selection and control of optical transitions. For silicon, shallow donors such as $\mathrm{P}$ and $\mathrm{Bi}$ have been shown to have long spin lifetimes $[5,8]$ and, thus far, have received

Published by the American Physical Society under the terms of the Creative Commons Attribution 3.0 License. Further distribution of this work must maintain attribution to the author(s) and the published article's title, journal citation, and DOI. the most attention for applications taking advantage of the spin degrees of freedom. However, acceptor centers offer strong potential advantages, and, indeed, combinations of donors and acceptors may also be attractive. Acceptors exhibit both strong spin-orbit coupling and long spin lifetime because symmetry removes the contact of the wave function with the (fluctuating) nuclear spins [13]; in addition, the orbital hyperfine interaction can be controlled, e.g., with strain [14]. Spin-orbit coupling is an important ingredient because it allows optical excitations that regulate the spin state via a selection rule; spin-orbit coupling is strongest in the valence band.

The major exception to the simple scaling from free atoms is the excited-state relaxation rate, which is due to phonon emission rather than photon emission as for atoms in free space, and is therefore much more difficult to predict theoretically. Donors show long lifetimes for orbital excited states [4,15-18], but for acceptors it is generally assumed that relaxation is very fast; e.g., for boron in diamond [19], relaxation after Lyman transitions (within the $p_{3 / 2}$ series of shallow acceptor states) is so fast that it has never been measured directly. The silicon-acceptor relaxation lifetime is unknown but bounded from below by the inverse linewidth of absorption spectra $[16,17]$, as was the case for shallow donors until recent time-resolved experiments with the pump-probe technique [15]. It has been shown that very careful sample preparation with isotopic purification and elimination of strain can produce long acceptor spin lifetimes measurable by magnetic 

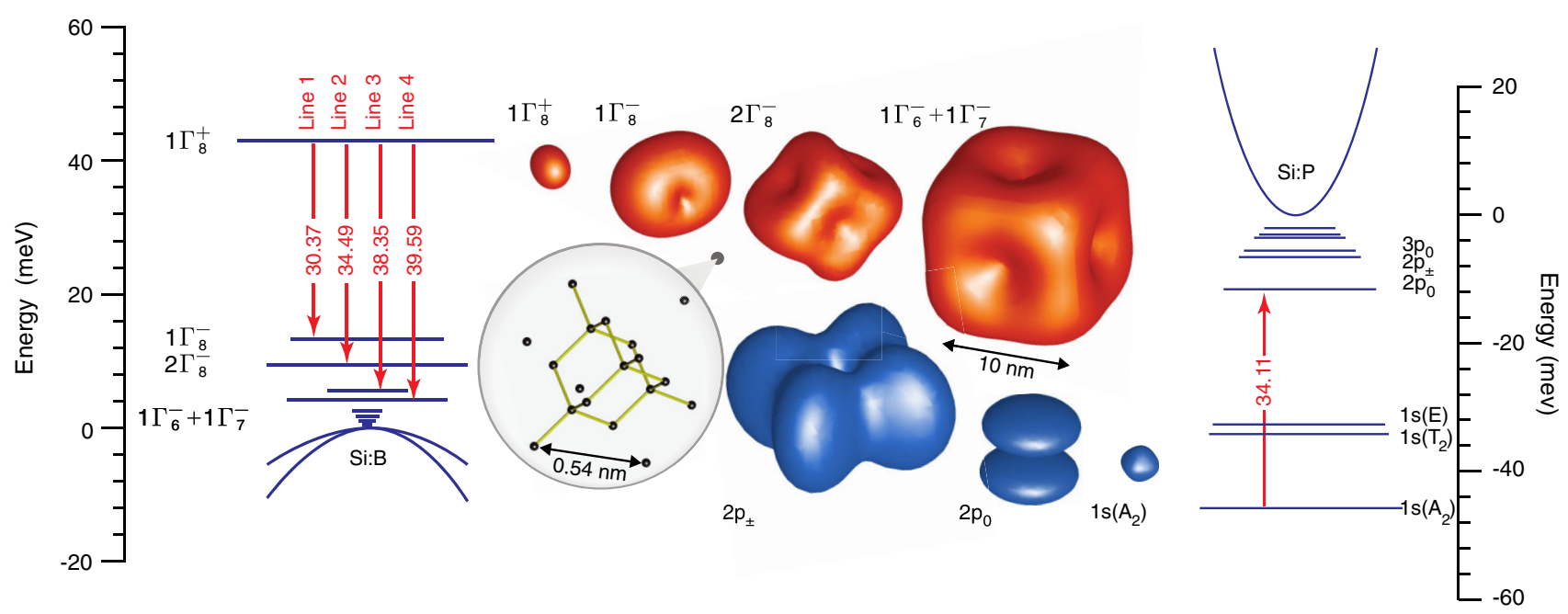

FIG. 1. Schematic of the energy levels and optical transitions in Si:B shallow acceptors. The acceptors Si:Al and Si:Ga have similar spectra with different chemical shifts for the ground state (the absorption spectra for these three species are shown in Fig. 7 below). The states are labeled with their symmetry from the $T_{d}$ group. The envelope functions for the lowest four states are shown (the surfaces are constant probability density surfaces that enclose $50 \%$ of the normalization). Each state is oriented relative to the cubic-crystal axes (indicated by the crystal-structure inset, which also illustrates the huge scale of the envelope functions relative to the lattice constant), and all are multiply degenerate. For each energy level, one of the cubic harmonics is shown. For comparison, the Si:P shallow donor wave functions are shown on the same scale; the dimensions are shown in $\mathrm{nm}$. The picture shows that the boron excited-state wave functions are somewhat larger than the excited phosphorus states.

resonance spectroscopy [20] and very sharp orbital transitions [16,17], but the linewidths of the lowest energy transitions remain an order of magnitude wider than for donors [17]. The main effect of random strain (or applied stress) is to remove the degeneracy of the light- and heavyhole bands, which splits the acceptor bound states and introduces inhomogeneous broadening. This effect is very important in frequency-domain spin resonance [20] and spectroscopy of orbital transitions [16,17], but, in the time domain, we eliminate all contributions to the inhomogeneous broadening (except those derived from different lifetimes associated with different local defect environments).

Effective-mass calculations of the wave functions have been shown to be useful in predicting that donor dynamics and experiments on $\mathrm{P}$ and As in silicon are consistent with single-phonon emission [4,15], though the established theoretical picture involves cascade relaxation via intermediate, very short-lived, dark states $[10,18]$. In this paper, we investigate, using time-domain spectroscopy with a free-electron laser, the orbital relaxation and dephasing in acceptors $\mathrm{B}, \mathrm{Al}$, and $\mathrm{Ga}$, and the donor Sb. Our measured decay rates for a variety of transitions, and those previously measured for $\mathrm{P}$ and As $[4,15]$, seem to scale simply with the phonon density of states, consistent with direct phonon relaxation to the ground state. In addition, these decay rates lead us to speculate that engineering the phonon density of states in nanostructures [21] offers the possibility of making the excited-state lifetimes much longer, with a maximum then determined by the radiative lifetime which is theoretically in the ns $-\mu$ s range.

\section{EXPERIMENTAL PROCEDURE}

To perform the time-resolved, energy-relaxation experiments, we used a "null method" variant of the standard pump-probe geometry, in which a third beam is used as a reference to balance out shot noise and drift [22-24]. Figure 2 is a schematic diagram of our experiment.

The free-electron laser, FELIX, in Nieuwegein (Netherlands), provided the required intense, picosecond pulses in the terahertz region of interest [22]. The laser pulses came in bursts of duration $7 \mu \mathrm{s}$ and repetition rate $5 \mathrm{~Hz}$. Each burst contained a train of micropulses of approximately $10 \mathrm{ps}$ duration and $25 \mathrm{MHz}$ repetition rate. The laser is easily and continuously tunable, and its center wavelength was tuned to the absorption line in

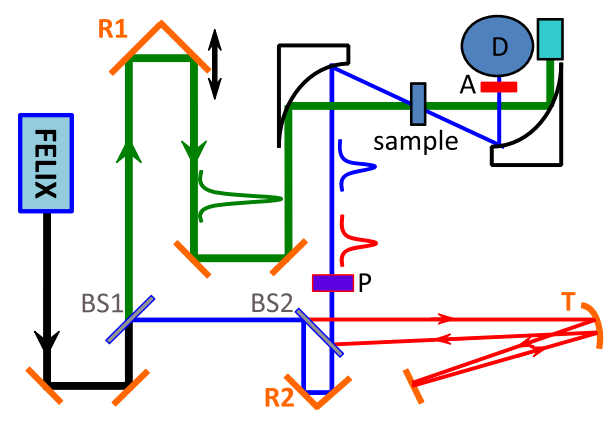

FIG. 2. The apparatus. 
each case. The pulses are approximately bandwidth limited with a Gaussian profile, and the pulse duration, which is controllable, was chosen to be approximately $10 \mathrm{ps}$ (corresponding to a spectral width of about $0.5 \%$ at a photon energy of $30 \mathrm{meV}$ ). We split the laser beam into two parts using a polypropylene pellicle beam splitter (BS1). The transmitted beam-the pump (green)traveled down an optical path with controllable length (R1) to provide a variable arrival time. The probe beam was further split into two identical, collinear pulses separated in time by half of the micropulse repetition time: 20 ns. This splitting was achieved with a second beam splitter (BS2), a second fixed retroreflector (R2) - which inverted the beam spatially — and a long inverting telescope arrangement (T) with magnification of 1 . This arrangement ensured that both beams undergo one reflection and one transmission from BS2 (so the intensities were exactly matched) and that any pulse-to-pulse fluctuations in the spatial profile were also matched (the main differences being due to different scattering by differing mirror imperfections and a small uncompensated divergence in the short, fixed path; these differences were several orders of magnitude smaller than the pulse-to-pulse fluctuations). The first of these two pulses acted as the probe (blue), and it arrived at the sample within the range of arrival times of the pump. The second pulse arrived long after the pump, at a time much greater than any of the relaxation or recombination times under investigation, and it acted as a reference (red). The spatially separated pump and probe and reference beams were reflected from a large, goldcoated, off-axis parabolic mirror to a focus on the sample, which was held in vacuum on the cold finger of a heliumflow cryostat with polypropylene film windows. The focused laser-beam spot size was measured (by investigating passage through a variety of fixed-diameter apertures) to be $4.5 \mathrm{~mm}$ (the aperture diameter admitting half the power). The angular separation of the beams at the sample was about $5^{\circ}$. The emerging pump and probe and reference beams were collected on a second off-axis parabolic mirror and collimated before refocusing on the detector (refocusing mirror not shown in Fig. 2).

To minimize the effects of water-vapor absorption in the atmosphere, all beams were transported in vacuum or in a dry-nitrogen filled tank, from which they exited via a polypropylene window. The focusing mirror, cryostat, and detector were outside the tank in a dry-nitrogen purged tent, with an approximately $40 \mathrm{~cm}$ path from the window to the sample and the same from the sample to the detector.

The micropulses had energy up to $\sim 1 \mu \mathrm{J}$ measured with a calibrated pyroelectric power meter. The intensity of the beams was independently controlled by sets of calibrated wire-grid attenuators (not shown). The electric-field polarization of the laser beam was always linear. In the case of the pump, the polarization was vertical, while in the case of the probe and reference beams, it was rotated by $90^{\circ}$ to horizontal by a polarization rotator consisting of three mirrors $(\mathrm{P})$. An analyzer polarizer (A) consisting of a freestanding wire mesh was placed before the detector (D) in order to eliminate scattered pump light entering the detector.

In all experiments, the detector used was a liquid-helium cooled Ge:Ga detector. The detector was biased alternately forwards and backwards at a frequency of $25 \mathrm{MHz}$ with a phase-locked circuit so that the probe arrived during forward-bias conditions and the (identical) reference arrived during reverse bias. The response time of the amplifier circuit was designed to lie between the pulse repetition time of $40 \mathrm{~ns}$ and the burst duration of $7 \mu \mathrm{s}$, so the detector gave a response of zero while the probe and reference were balanced. The effect of the pump pulse was to reduce the absorption of the probe (depending on the delay between them); this effect unbalanced the detector, and a response was produced. The signals measured were therefore all averaged over the burst and further boxcar averaged over several (usually five) macropulses. To extract the dynamics, the change in absorption was measured as a function of the time delay between the pump and the probe. In all cases described below, scans (of delay, intensity, etc.) were repeated and averaged.

To investigate the importance of heating effects, we also used a pulse-slicing technique to switch out an individual micropulse from each burst. In this case, a semiconductor (silicon) slab and retroreflector were inserted into the beam upstream of BS1. The slab was transparent to the THz light from FELIX, but when the slab was illuminated with a synchronized Nd:YAG laser pulse, it became highly reflective; any part of the beam that was between the mirror and slab at the instant the YAG pulse hit was reflected out (and the other pulses were rejected). We arranged for a single FELIX pulse to be reflected out, directed through the sample, and we repeated the experiments. Apart from the need for much longer acquisition times, no changes in the measured lifetimes were observed. All data shown in this paper were taken with the full macropulse.

To measure the dephasing time for coherent states, we used the photon-echo technique [4], which is the orbital equivalent of the microwave-spin echo used in nuclear magnetic resonance (NMR). The setup was the same as for the pump-probe experiment, Fig. 2, except that the reference arm was removed (BS2 was replaced by a mirror). The polarization rotator $\mathrm{P}$ was set so that the beams were all vertically polarized, and the attenuators were set so that the second pulse was about 4 times more intense than the first (i.e., the electric field was twice as much). No attempt was made to calibrate the absolute pulse amplitude because echoes are produced for almost any valuenevertheless, we will refer to the first pulse as the " $\pi / 2$ " pulse, and to the second, the " $\pi$ " pulse. The detector was translated to detect the emission of the echo, which appears in the phase-matching direction $2 \mathbf{k}_{2}-\mathbf{k}_{1}$, where $\mathbf{k}_{1}$ and $\mathbf{k}_{2}$ 
are the propagation directions of the first and second pulses, respectively. In this direction, significant light was only detected when both laser beams were present; i.e., negligible light was detected with either one of the laser beams blocked. With such a setup, it has previously been shown [4] that the light detected is proportional to the square of the coherent polarization remaining in the sample at the echo emission time, which is $2 t$ after the coherence is first created by the first laser pulse, where $t$ is the time delay between the pulses. The signal therefore decays with time constant $T_{2} / 4$, where $T_{2}$ is the phase-coherence lifetime.

We investigated two boron-doped samples, produced using different growth methods: float-zone $(\mathrm{Fz})$ and Czochralski $(\mathrm{Cz})$ growths. The $\mathrm{Cz}$ wafer had a thickness of $200 \mu \mathrm{m}$ doped with B to yield a room-temperature resistivity of $\rho=5 \Omega \mathrm{cm}$, which corresponds to an acceptor density of approximately $2.7 \times 10^{15} \mathrm{~cm}^{-3}$. The Fz sample was $3 \mathrm{~mm}$ thick, cut from a single Fz-Si crystal doped with B having $\rho$ between 75 and $125 \Omega \mathrm{cm}$ (approximately $1.3 \times 10^{14} \mathrm{~cm}^{-3}$ ). The Al-, Ga-, and $\mathrm{Sb}$-doped samples were cut from single $\mathrm{Cz}$-Si crystals of $5 \Omega \mathrm{cm}$ with a thickness of $500 \mu \mathrm{m}$. No special polishing was found to be necessary, given that the wavelengths used are several tens of microns and sample roughness is usually found to be beneficial in reducing Fabry-Perot effects, equivalent to multiple reflected pulses in the time domain.

\section{RATE-EQUATION MODEL}

To explain the dynamics, we use a rate-equation model, previously developed for shallow donors in silicon [15]. At its simplest, this model includes transitions between three levels: the ground state, the excited state (being pumped by the laser pulse), and the continuum of traveling wave states at higher energies (in this case, the valence band). In this way, the rate-equation model takes account of both the photo-pumping and subsequent relaxation back to the ground state, and the photoionization of holes from the excited state into the continuum and subsequent recombination with the ionized acceptors. The rate equations are

$$
\begin{aligned}
\frac{d p_{g}}{d t} & =\frac{p_{x}}{T_{1}}+\frac{1}{T_{\mathrm{recom}}^{\mathrm{GS}}} p^{2}, \\
\frac{d p_{x}}{d t} & =-\frac{p_{x}}{T_{1}}+\frac{1}{T_{\mathrm{recom}}^{\mathrm{EX}}} p^{2}, \\
\frac{d p}{d t} & =-\left[\frac{1}{T_{\text {recom }}^{\mathrm{GS}}}+\frac{1}{T_{\text {recom }}^{\mathrm{EX}}}\right] p^{2},
\end{aligned}
$$

where $p_{g}$ and $p_{x}$ are the dimensionless occupation probabilities of the acceptor-center ground states and excited states, respectively. The probability of holes occupying the valence band is $p$, which, by charge conservation, is equal to the probability of acceptor ionization, and $p+p_{g}+p_{x}=1$. The relaxation rate from the excited state to the ground state is $1 / T_{1}$. The recombination yield is proportional to the product of the numbers of free holes and ionized acceptors (i.e., to $p^{2}$ ), and $1 / T_{\text {recom }}^{\mathrm{GS}}$ and $1 / T_{\mathrm{recom}}^{\mathrm{EX}}$ are the recombination probabilities per unit time of free holes into the ground and excited states, respectively.

In the experiment, the pump produces initial populations $p_{g 0}, p_{x 0}$, and $p_{0}$ (for the ground state, excited state, and ion and free-hole occupation probabilities). The probe photons can produce absorption by atoms in their ground state and stimulated emission by atoms in their excited state, with the same cross section. The net absorption is therefore proportional to the difference in the excited- and groundstate populations, and the measured-probe absorption is proportional to $p_{g}(t)-p_{x}(t)$. Equations (1) can be solved analytically to give

$$
p_{g}(t)-p_{x}(t)=p_{g 0}+p_{x 0}\left[1-2 e^{-\gamma t}\right]+p_{0}[a(t)-2 b(t)],
$$

where $\gamma=1 / T_{1}$ and

$$
\begin{aligned}
a(t)= & \left(\frac{t}{t+t_{R}}\right), \\
b(t)= & \left(\frac{T_{\mathrm{recom}}^{\mathrm{tot}}}{T_{\mathrm{recom}}^{\mathrm{EX}}}\right)\left\{a(t)+e^{-\gamma t}-1\right. \\
& \left.+\gamma t_{R} e^{-\gamma\left(t+t_{R}\right)}\left[E_{1}\left(-\gamma t_{R}\right)-E_{1}\left(-\gamma\left(t+t_{R}\right)\right)\right]\right\} .
\end{aligned}
$$

In this treatment, $E_{1}(z)$ is the exponential integral $\int_{z}^{\infty} \frac{e^{-t}}{t} d t$, the initial ion recombination time is $t_{R}=T_{\text {recom }}^{\text {tot }} / p_{0}$, and $1 / T_{\text {recom }}^{\text {tot }}=1 / T_{\text {recom }}^{\mathrm{GS}}+1 / T_{\text {recom }}^{\mathrm{EX}}$. It is clear that Rydbergstate relaxation, the second term in (2), gives rise to exponential components in the transient absorption with lifetime $T_{1}$. Recombination, which follows multiphoton ionization and is produced by the third term in Eq. (2), gives rise to reciprocal decay (i.e., of the form $1 / y=$ $\left.t / T_{\text {recom }}^{\text {tot }}+1 / y_{0}\right)$ characterized by a time scale $T_{\text {recom }}^{\text {tot }}$.

The model can be extended to include intermediate states if they are important. In the limit that ionization is negligible and relaxation to an intermediate state is dominant, we have

$$
\frac{d p_{g}}{d t}=\frac{p_{i}}{T_{g}}, \quad \frac{d p_{i}}{d t}=-\frac{p_{i}}{T_{g}}+\frac{p_{x}}{T_{1}}, \quad \frac{d p_{x}}{d t}=-\frac{p_{x}}{T_{1}},
$$

where $p_{i}$ is the population of the intermediate state (or states) and $T_{g}$ is the relaxation time associated with refilling the ground state (and $T_{1}$ is the relaxation time of the excited state as before, but now associated with transitions to the intermediate state). The solution (assuming $T_{1} \neq T_{g}$ ) is

$$
\begin{aligned}
p_{g}(t)-p_{x}(t)= & 1-\frac{p_{x 0}}{T_{g}-T_{1}}\left[T_{g} \exp \left(-t / T_{g}\right)\right. \\
& \left.+\left(T_{g}-2 T_{1}\right) \exp \left(-t / T_{1}\right)\right] .
\end{aligned}
$$


If $\quad T_{1} \gg T_{g}, \quad$ then $\quad p_{g}-p_{x} \approx 1-2 p_{x 0} \exp \left(-t / T_{1}\right)$; i.e., only the slower process is measurable. However, if $T_{1} \ll T_{g}, \quad$ then $\quad p_{g}-p_{x} \approx 1-p_{x 0}\left[\exp \left(-t / T_{g}\right)+\right.$ $\left.\exp \left(-t / T_{1}\right)\right]$; i.e., the transient absorption experiment is equally sensitive to both the emptying of the excited state and the refilling of the ground state.

\section{RESULTS}

For acceptor centers in silicon, there are four main optical transitions which dominate the spectrum. The states are labeled according to their symmetry [12,16,17,25], as shown in Fig. 1 (spectra are shown in Fig. 7 below). Figure 3 shows the measured transient transmission for line 1 at $30.37 \mathrm{meV}$ of a $\mathrm{Si}: \mathrm{B}$ sample with different pump fluences (on both log-linear and reciprocal-linear axes, for reasons that will become clear in the discussion below). At low pumping powers, the decay of Fig. 3 has two components. At higher pumping power, the fast component disappears, but the slow component remains. The results for the $\mathrm{Cz}$ sample are similar. This is in strong contrast to the many-electron situation for quantum dots, where increasing the intensity introduces faster electron-electron processes.

For many other acceptor transitions, e.g., other Si:B lines, and the $\mathrm{Si}: \mathrm{Al}$ transition line 1 at $54.89 \mathrm{meV}$ [the latter is shown in Fig. 4(a)], the situation is similar; they also have two components, and again the fast component

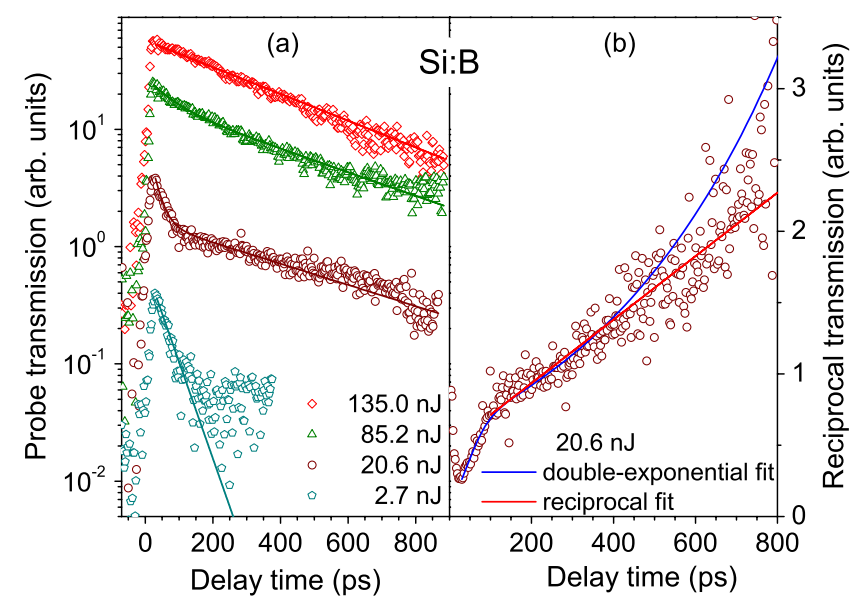

FIG. 3. Pump-probe transients for Si:B line 1. (a) The change in probe transmission induced by the pump as a function of the time delay between pump and probe pulses, observed in the Fz Si:B sample at $5 \mathrm{~K}$ with photon energy of $30.37 \mathrm{meV}$. The rise of the leading edge indicates the pulse duration, which was approximately $10 \mathrm{ps}$. The lowest pump pulse energy $(2.7 \mathrm{~nJ})$ corresponds to a focused photon fluence of $\approx 1.2 \times$ $10^{17}$ photons $\mathrm{m}^{-2}$. Also shown are fits using a doubleexponential decay. (b) The same data as in (a) for $20.6 \mathrm{~nJ}$ but plotting the reciprocal of the transmission. The same fitted double-exponential decay from (a) is shown (blue line), along with a reciprocal decay fit (red line). We do not provide the parameters that determine the ordinate scale for either fit because that axis is in arbitrary units.

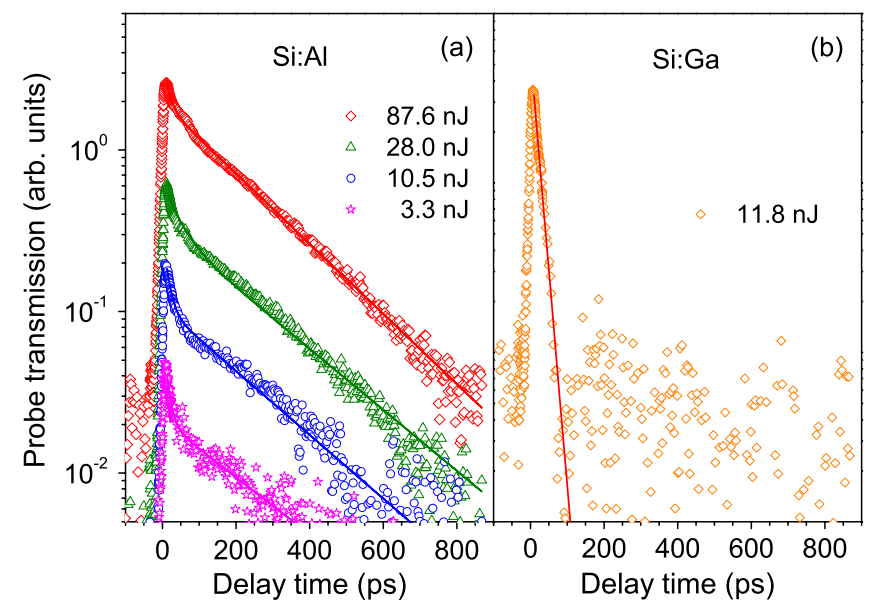

FIG. 4. Same as Fig. 3(a) for Si:Al and Si:Ga samples at $5 \mathrm{~K}$. (a) Optical transition line 1 for $\mathrm{Si}: \mathrm{Al}$, and (b) optical transition line 1 for Si:Ga.

disappears at higher pumping powers. Line 1 at $58.24 \mathrm{meV}$ for the Ga-doped Si sample [Fig. 4(b)] is different. There is only a single, fast component whose decay rate is below the resolution determined by the pulse duration (this lower limit is approximately $10 \mathrm{ps}$ ), and unlike the other acceptors, no slow component of the decay appeared when the pulse energy was raised.

We also report similar experiments on the donor species $\mathrm{Sb}$ (Fig. 5), to augment previous data for the lifetimes for $\mathrm{P}$ and As [15]. As for the other donors, we observe a singlecomponent decay.

For the data of Fig. 3(a), we see that a fit with an exponential decay [i.e., of the form $\exp (-\gamma t)$ according to Eqs. (2) or (4)] adequately describes the initial fast component, but Fig. 3(b) shows that the slow component seems to imply a reciprocal decay [i.e., of the form $1 / y=$ $t / T_{\text {recom }}^{\text {tot }}+1 / y_{0}$ according to Eq. (2)]. Figure 3(b) shows an example comparison of the fits for the long component, and the r.m.s. residual for the exponential fit is just over

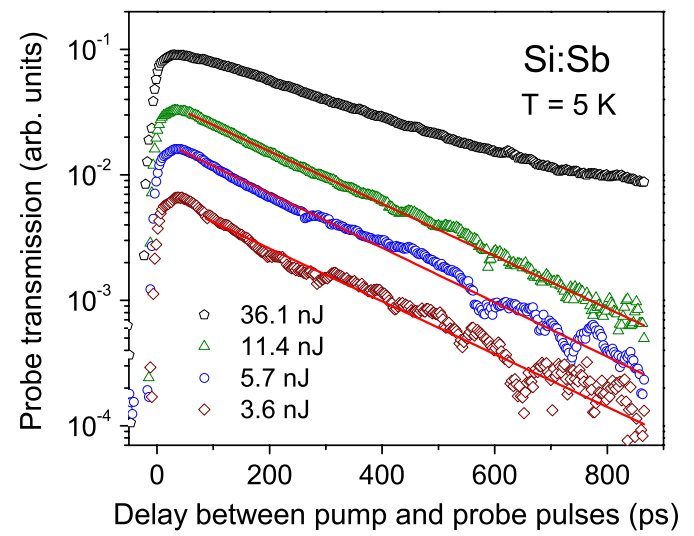

FIG. 5. Same as Fig. 3 for the $1 s-2 p_{0}$ transition in $\mathrm{Si}: \mathrm{Sb}$ at $5 \mathrm{~K}$. Also shown are fits using a single-component decay (unlike the acceptors which have two-component decays). 
double that for the reciprocal fit. We conclude that the fast component observed at low intensity is due to a relaxation process, while the slow component observed at high intensity is due to the recombination. In contrast, for the donor centers in silicon [15], the ionization caused by multiphoton absorption during pumping is unimportant, meaning that the photoionization cross section for the excited states is much lower than for those associated with acceptor centers.

We used exponential fits to the fast components (most prominent at low intensity) and reciprocal fits to the slow components (dominating at high intensity). For the Si:B line 1 , our fits yield time constants for the relaxation of $57 \pm 10 \mathrm{ps}$ and for the recombination $T_{\text {recom }}^{\text {tot }}=440 \mathrm{ps} \pm$ $10 \%$, and higher-energy transitions (lines 2, 3, and 4) have successively reduced relaxation time but similar $T_{\text {recom }}^{\text {tot }}$. For Si:Al line 1, which is at higher transition energies, we find a relaxation time of $33 \pm 5 \mathrm{ps}$ and $T_{\text {recom }}^{\text {tot }}=250 \mathrm{ps} \pm$ $10 \%$. Continuing this trend to higher energies, the $\mathrm{Si}: \mathrm{Ga}$ sample line 1 and line 2 of the Si:Al sample exhibit a decay time below the resolution determined by the pulse duration (and unlike the other transitions, no slow component of the decay appeared when the pulse energy was raised). We do not provide the parameters that determine the ordinate scale (e.g., $y_{0}$ ) for any fit because the transmission axis is in arbitrary units.

The photon-echo experiment results for the Si:B transition line 1 are shown in Fig. 6. As mentioned above, the echo signal as a function of the delay, $t$, between the $\pi / 2$ and $\pi$ pulses decays exponentially with time constant $T_{2} / 4$, and the resulting $T_{2}$ was $69 \mathrm{ps}$. The error from a least-squares fit was 2 ps. For all other transitions investigated, the pulse duration was longer than the upper limit for the time constant given by $T_{1} / 2$ (for lines that are homogeneously broadened with no sources of dephasing other than the population decay, $T_{2}=2 T_{1}$ ), so we were not able to verify whether or not additional sources of

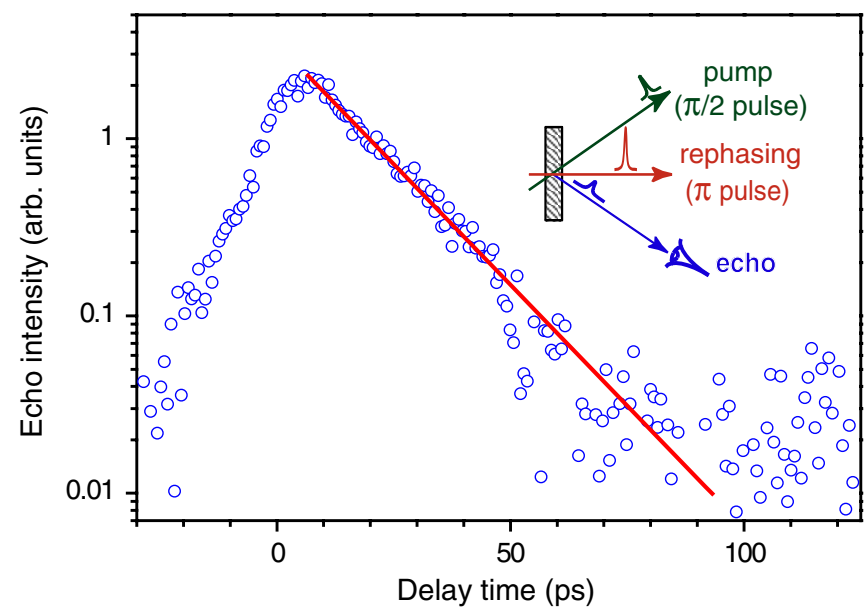

FIG. 6. Photon echo for Si:B transition line 1. The abscissa is the time between the $\pi / 2$ and $\pi$ pulses. The inset shows a schematic of the beam geometry. dephasing are important in any other cases. For the data of Fig. 6, the rise time is 7 ps.

\section{DISCUSSION}

The photon-echo result for $T_{2}$ in line 1 for Si:B is very similar to the population relaxation time measured from the pump-probe experiment (within the errors of the exponential fit). We conclude that the time measured is $T_{1}$, the emptying time of the excited state, and that, as for donors [4], the silicon crystal acts analogously to an "atom trap" in the sense that it adds no extra sources of decoherence other than the population decay. It seems reasonable to suppose that the same is true of other lines and other acceptors (and donors), so long as the sample temperature and photoexcited electron density are comparable (because phonons and itinerant electrons would be the sources of homogeneous broadening). There is an optically dark, Raman-allowed state $23 \mathrm{meV}$ above the ground state [12] (i.e., below all of the optically allowed excited states). If relaxation is via a cascade through this state, it must be extremely short-lived.

Figure 7 shows the measured decay times of the fast component as a function of photon energy for the Si:B, $\mathrm{Si}: \mathrm{Al}$, and $\mathrm{Si}: \mathrm{Ga}$ samples. Superposed are the absorption spectra as measured by Fourier transform infrared spectroscopy (FTIR) with a resolution of $0.02 \mathrm{meV}$.

To understand the origin of the population relaxation of the acceptor centers in silicon, we first consider the possibility of direct relaxation from the excited state to the ground state by emission of a single phonon. Following [26], an approximate expression for the acoustical phonon emission rate via the deformation potential, at zero temperature, is

$$
W=\frac{\pi E_{1}^{2} \omega}{\rho c_{s}^{2}}|\langle f|\exp i \mathbf{q} \cdot \mathbf{r}| i\rangle|^{2} g(\hbar \omega),
$$

where $\omega, \mathbf{q}$ are the phonon frequency and wave vector, $g(\hbar \omega)$ is the density of phonon states, and $E_{1}, c_{s}$, and $\rho$ are, respectively, the deformation potential, the speed of sound, and the mass density of Si. The one-phonon density of states in silicon [27] is shown in Fig. 7(d). The result of Eq. (5) for boron in the case of $2 \Gamma_{8}^{-}$to $1 \Gamma_{8}^{+}$, i.e., line 1 , produces $2 \pi / W=90 \mathrm{ps}$, which is in reasonable agreement with the experiment. As can be seen from Fig. 7(a), the lifetime monotonically decreases as the frequency increases, which is in agreement with the approximation of Eq. (5), so long as the matrix element does not vary dramatically from transition to transition. The shortest lifetimes on Fig. 7 are clearly associated with the highest density of phonon states corresponding to the TO phonon band, while the longest lifetimes are associated with the smallest density of phonon states corresponding to the tail of the LA band, as shown in Fig. 8. Thus, it appears that our data for the bound-to-bound relaxation decay are consistent with single-phonon emission without 


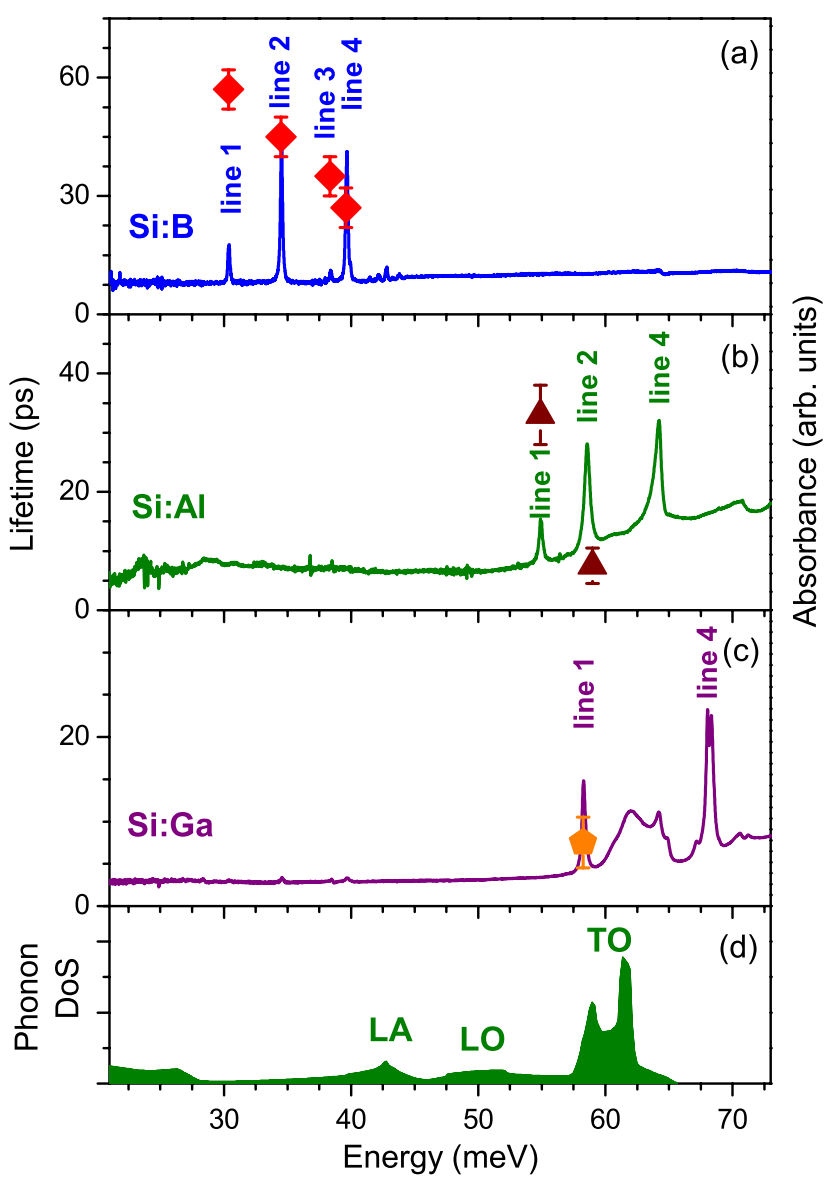

FIG. 7. Spectral dependence of acceptor population decay. (a) The absorption spectrum for B-doped $\mathrm{Si}$ at $5 \mathrm{~K}$, measured by FTIR spectroscopy with $0.02 \mathrm{meV}$ resolution (right axis). The decay time of the indicated transitions at low laser power, determined from pump-probe transients (such as those in Fig. 3) are shown by the solid symbols (left axis). (b) The corresponding results for Si:Al. (c) The corresponding results for Si:Ga. (d) The one-phonon density of states (DOS), including longitudinal-optical (LO), longitudinal-acoustic (LA), and transverse-optical (TO) phonon modes of silicon, which determines the phonon-emission decay rate at low temperatures (taken from Ref. [27]).

intermediate states. A similar trend with density of phonon states at the ground-state binding energy explains the variation in bound-free recombination rates, and we suppose that they, too, are determined by single-phonon emission from the continuum to the ground state.

The situation with donors is more complex, and there are a number of theoretical studies of the relaxation that have indicated that the dominant relaxation pathway is via intermediate dark states-the valley-orbit split components of the ground state $[10,18]$. Along with the acceptor transitions, Fig. 8 also shows the measured rates from timeresolved experiments for donor transitions, which also appear to follow the one-phonon density of states, but with a proportionality constant that is about 4 times

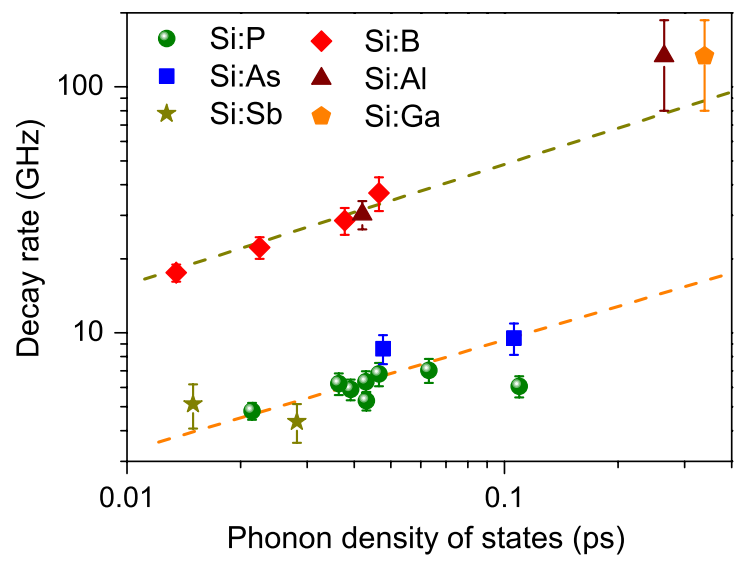

FIG. 8. Universal relationship between population decay and one-phonon density of states. The phonon DOS is from Fig. 7(d) and given in units of inverse frequency [27]. The decay rates, $1 / T_{1}$, at $5 \mathrm{~K}$ are shown for the acceptors from Fig. 7, along with donors-phosphorus and arsenic from Ref [15] and antimony from Fig. 5. Two acceptor transitions are near the TO phonon band and have very fast decays at the limit of our resolution. The lines shown are guides to the eye with slope of 1 (i.e., proportional relationships).

smaller. Included among the donor data are the lifetimes for $\mathrm{P}$ and As previously reported [15] and our new results for $\mathrm{Sb}$. Clearly, the fact that the relaxation transitions follow a simple relationship irrespective of the starting state suggests that, to within experimental error, the precise details of the envelope wave function are not important. However, the density of final states plays a role: While the final state of the decay for shallow donors in silicon is twofold degenerate on account of spin, shallow-acceptor ground states show fourfold degeneracy.

For a simple two-level exponential population decay with decay constant $T_{1}$, the Fourier transform produces a Lorentzian line with full width at half maximum of $2 \pi \Delta \nu_{\text {FWHM }}=1 / T_{1}$. Natural silicon samples exhibit strong inhomogeneous broadening due to impurities, defects, alloying, and strain so that their linewidths are much greater than this intrinsic minimum [12,26,28], though isotopically pure silicon has been shown to produce much sharper lines $[16,17,20]$ that are close to ideal. The linewidths from the absorption spectrum (FTIR) of isotopically pure material [17] for $\mathrm{Si}: \mathrm{P}$ and $\mathrm{Si}: \mathrm{B}$, and inverse lifetimes from the pump-probe experiments (this work and Ref. [15]) are shown in Fig. 9. We also show the phonon DOS, which correlates well with the donor rates from both measurement techniques, but the acceptor linewidths have a very different behavior.

Further theory is needed to validate a picture of onephonon decay, which suggests a method for engineering orbital decay rates. Time-resolved experiments on the high-energy states of isotopically enriched Si:B would be attractive because of the very sharp lines, but it is not trivial to produce FELIX pulses appropriate for measurement of 


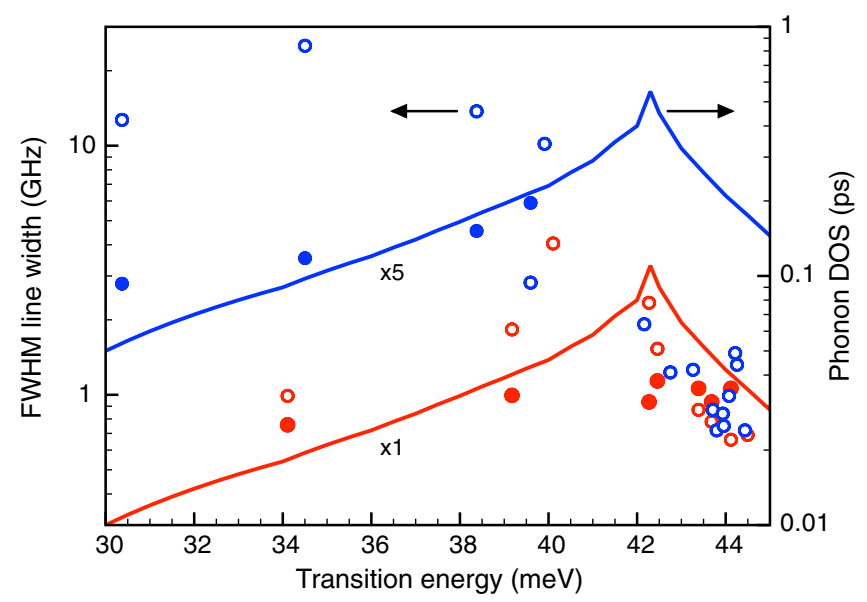

FIG. 9. The FWHM linewidth from the FTIR of isotopically pure ${ }^{28} \mathrm{Si}$ from Ref. [17] (open symbols) and inverse lifetime $1 / 2 \pi T_{1}$ at low pumping power from this work and Ref. [15] (solid symbols) for various $\mathrm{Si}: \mathrm{P}$ (red) and $\mathrm{Si}: \mathrm{B}$ (blue) transitions versus transition energy. In the case of the Si:B FTIR, only transitions that are strong and well isolated from other transitions have been included here. The phonon DOS (solid lines) has been scaled by the values indicated on the figure.

the higher-energy transitions; this must therefore be the subject of future work.

There is considerable work on the dynamics of spin and energy relaxation in quantum dots, which are also artificial atoms. Usually, electrons and holes are created together in an exciton and are not independent, so hole relaxation in undoped dots is very fast (e.g., subpicoseconds to few picoseconds in colloidal nanocrystals [29]) because of the extra degree of freedom provided by the electron. Meanwhile, in doped dots, hole relaxation is nearly as fast (e.g., few ps in p-type InAs dots [30]) but depends on the level separation relative to the various phonon energies. We note that donor potentials are more reproducible than for quantum dots, and there are many applications for which this is beneficial. The only other information on excited-state dynamics for acceptors is for GaAs; the transverse-spin dephasing time for $\mathrm{Mn}$ acceptors is of the order of $400 \mathrm{ps}$ at helium temperatures [31], and, similarly, the orbital relaxation time for $\mathrm{Be}$ is $350 \mathrm{ps}$ [32]. It seems likely that, in the latter case, given the similarity of the lifetimes with the long component in our data, it is the recombination of ionized acceptors that is being measured rather than the intra-acceptor lifetime.

\section{CONCLUSION}

We show that the population relaxation times of the antihydrogenic optical transitions in natural (isotopically mixed) $p$-Si samples are in the range of 10 to $60 \mathrm{ps}$. The maximum relaxation time corresponds to approximately 400 optical cycles (i.e., Q is approximately 400 ), which is only about 3 times shorter than the maximum for $n$-type systems. The lifetimes follow a simple universal relationship with the phonon density of states and the final state multiplicity, irrespective of the details of the envelope function and its symmetry. The surprising longevity of the acceptor states in natural silicon is totally unanticipated from frequency-domain spectroscopy, and it also shows that orbital-state lifetimes are affected by very different processes than spin-state lifetimes for the orbital ground state, which, according to magnetic resonance data, are much shorter for acceptors compared to donors. Beyond demonstrating that we can do the physics of antihydrogen in natural silicon, our data show that $\mathrm{THz}$ manipulation of acceptor states could underpin novel devices for this underexploited frequency domain. We have added coherent control of acceptors to the toolkit for nano-orbitronics and spintronics. The combination of acceptors and donors will allow fabrication of multispecies molecules (e.g., dimers of $\mathrm{P}-\mathrm{Sb}$ or $\mathrm{P}-\mathrm{B}$, and trimers such as Bi-P-Bi, etc.), trapped permanently in the host crystal, potentially fabricated with arbitrary spacing and symmetry using the atomic-scale lithography techniques being developed [7]; this idea will open up new possibilities for artificial molecules in the solid state.

\section{ACKNOWLEDGMENTS}

We gratefully acknowledge the support of the EPSRCUK (COMPASSS, Grant No. EP/H026622/1) and the FOM-NL in providing the required beam time on FELIX. We also highly appreciate the skillful assistance by the FELIX staff. We are grateful to M. L. Y. Pang for artwork in Fig. 1. We also acknowledge helpful discussions with Professor M. Thewalt.

[1] N. Piro, F. Rohde, C. Schuck, M. Almendros, J. Huwer, J. Ghosh, A. Haase, M. Hennrich, F. Dubin, and J. Eschner, Heralded Single-Photon Absorption by a Single Atom, Nat. Phys. 7, 17 (2010).

[2] M. Hosseini, G. Campbell, B. M. Sparkes, P. K. Lam, and B.C. Buchler, Unconditional Room-Temperature Quantum Memory, Nat. Phys. 7, 794 (2011).

[3] A. M. Stoneham, A.J. Fisher, and P.T. Greenland, Optically Driven Silicon-Based Quantum Gates with Potential for High-Temperature Operation, J. Phys. Condens. Matter 15, L447 (2003).

[4] P. T. Greenland, S. A. Lynch, A. F. G. van der Meer, B. N. Murdin, C. R. Pidgeon, B. Redlich, N. Q. Vinh, and G. Aeppli, Coherent Control of Rydberg States in Silicon, Nature (London) 465, 1057 (2010).

[5] G.W. Morley, M. Warner, A. M. Stoneham, P. T. Greenland, J. van Tol, C. W. M. Kay, and G. Aeppli, The Initialization and Manipulation of Quantum Information Stored in Silicon by Bismuth Dopants, Nat. Mater. 9, 725 (2010).

[6] B. N. Murdin, Juerong Li, M. L. Y. Pang, E. T. Bowyer, K. L. Litvinenko, S. K. Clowes, H. Engelkamp, C.R. 
Pidgeon, I. Galbraith, N. V. Abrosimov, H. Riemann, S. G. Pavlov, H.-W. Hübers, and P. G. Murdin, Si:P as a Laboratory Analogue for Hydrogen on High Magnetic Field White Dwarf Stars, Nat. Commun. 4, 1469 (2013).

[7] P.M. Koenraad and M.E. Flatté, Single Dopants in Semiconductors, Nat. Mater. 10, 91 (2011).

[8] Alexei M. Tyryshkin, Shinichi Tojo, John J.L. Morton, Helge Riemann, Nikolai V. Abrosimov, Peter Becker, Hans-Joachim Pohl, Thomas Schenkel, Michael L. W. Thewalt, Kohei M. Itoh, and S. A. Lyon, Electron Spin Coherence Exceeding Seconds in High-Purity Silicon, Nat. Mater. 11, 143 (2011).

[9] R.H. Page, R. J. Beach, V. K. Kanz, and W. F. Krupke, Multimode-Diode-Pumped Gas (Alkali-Vapor) Laser, Opt. Lett. 31, 353 (2006).

[10] S. G. Pavlov, R. Eichholz, N. V. Abrosimov, B. Redlich, and H.W. Hubers, Multifrequency Terahertz Lasing from Codoped Silicon Crystals, Appl. Phys. Lett. 98, 061102 (2011).

[11] T. N. Adam, R. T. Troeger, S. K. Ray, P.-C. Lv, and J. Kolodzey, Terahertz Electroluminescence from BoronDoped Silicon Devices, Appl. Phys. Lett. 83, 1713 (2003).

[12] A. K. Ramdas and S. Rodriguez, Spectroscopy of the Solid-State Analogues of the Hydrogen Atom: Donors and Acceptors in Semiconductors, Rep. Prog. Phys. 44, 1297 (1981).

[13] D. Brunner, B. D. Gerardot, P. A. Dalgarno, G. Wüst, K. Karrai, N. G. Stoltz, P. M. Petroff, and R. J. Warburton, A Coherent Single-Hole Spin in a Semiconductor, Science 325, 70 (2009).

[14] F. Maier and D. Loss, Effect of Strain on HyperfineInduced Hole-Spin Decoherence in Quantum Dots, Phys. Rev. B 85, 195323 (2012).

[15] N. Q. Vinh, P. T. Greenland, K. Litvinenko, B. Redlich, A. F. G. van der Meer, S. A. Lynch, M. Warner, A. M. Stoneham, G. Aeppli, D. J. Paul, C.R. Pidgeon, and B. N. Murdin, Silicon as a Model Ion Trap: Time Domain Measurements of Donor Rydberg States, Proc. Natl. Acad. Sci. U.S.A. 105, 10649 (2008).

[16] D. Karaiskaj, J. A. H. Stoz, T. Meyer, M. L. W. Thewalt, and M. Cardona, Impurity Absorption Spectroscopy in ${ }^{28} \mathrm{Si}$ : The Importance of Inhomogeneous Isotope Broadening, Phys. Rev. Lett. 90, 186402 (2003).

[17] M. Steger, A. A. Yang, D. Karaiskaj, M. L. W. Thewalt, E. E. Haller, J. W. Ager, M. Cardona, H. Riemann, N. V. Abrosimov, A. V. Gusev, A. D. Bulanov, A. K. Kaliteevskii, O. N. Godisov, P. Becker, and H. J. Pohl, Shallow Impurity Absorption Spectroscopy in Isotopically Enriched Silicon, Phys. Rev. B 79, 205210 (2009).

[18] V. Tyuterev, J. Sjakste, and N. Vast, Theoretical Intrinsic Lifetime Limit of Shallow Donor States in Silicon, Phys. Rev. B 81, 245212 (2010).
[19] C. E. Nebel E. Rohrer, and M. Stutzmann, Long Living Excited States in Boron Doped Diamond, J. Appl. Phys. 89, 2237 (2001).

[20] R. Stegner, H. Tezuka, T. Andlauer, M. Stutzmann, M. L. W. Thewalt, M. S. Brandt, and K. M. Itoh, Isotope Effect on Electron Paramagnetic Resonance of Boron Acceptors in Silicon, Phys. Rev. B 82, 115213 (2010).

[21] R. Beardsley, A. V. Akimov, B. A. Glavin, W. Maryam, M. Henini, and A. J. Kent, Optical Detection of Folded MiniZone-Edge Coherent Acoustic Modes in a Doped GaAs/ AlAs Superlattice, Phys. Rev. B 82, 041302(R) (2010).

[22] B. N. Murdin, Far-Infrared Free-Electron Lasers and Their Applications, Contemp. Phys. 50, 391 (2009).

[23] P.C. Findley, C.R. Pidgeon, R. Kotitschke, A. Hollingworth, B. N. Murdin, C. J. G. M. Langerak, A. F. G. van der Meer, C. M. Ciesla, J. Oswald, A. Homer, G. Springholz, and G. Bauer, Auger Recombination Dynamics of Lead Salts under Picosecond Free-ElectronLaser Excitation, Phys. Rev. B 58, 12908 (1998).

[24] M. Califano, N. Q. Vinh, P. J. Phillips, Z. Ikonić, R. W. Kelsall, P. Harrison, C. R. Pidgeon, B. N. Murdin, D. J. Paul, P. Townsend, J. Zhang, I. M. Ross, and A. G. Cullis, Interwell Relaxation Times in $p$-Si/SiGe Asymmetric Quantum Well Structures: Role of Interface Roughness, Phys. Rev. B 75, 045338 (2007).

[25] R. Buczko and F. Bassani, Shallow Acceptor Resonant States in Si and Ge, Phys. Rev. B 45, 5838 (1992).

[26] F. Beleznay and G. Pataki, Remarks on the Recombination of Electrons and Donors in n-Type Germanium, physica status solidi (b) 13, 499 (1966).

[27] C. Flensburg and R. F. Stewart, Lattice Dynamical DebyeWaller Factor for Silicon, Phys. Rev. B 60, 284 (1999).

[28] S. A. Lynch, G. Matmon, S. G. Pavlov, K. L. Litvinenko, B. Redlich, A. F. G. van der Meer, N. V. Abrosimov, and H.-W. Hübers, Inhomogeneous Broadening of Phosphorus Donor Lines in the Far-Infrared Spectra of SingleCrystalline SiGe, Phys. Rev. B 82, 245206 (2010).

[29] R. R. Cooney, S. L. Sewall, K. E. H. Anderson, E. A. Dias, and P. Kambhampati, Breaking the Phonon Bottleneck for Holes in Semiconductor Quantum Dots, Phys. Rev. Lett. 98, 177403 (2007).

[30] J. Siegert, S. Marcinkevičius, and Q.X. Zhao, Carrier Dynamics in Modulation-Doped InAs/GaAs Quantum Dots, Phys. Rev. B 72, 085316 (2005).

[31] R. C. Myers, M. H. Mikkelsen, J. M. Tang, A. C. Gossard, M.E. Flatte, and D.D. Awschalom, Zerofield Optical Manipulation of Magnetic Ions in Semiconductors, Nat. Mater. 7, 203 (2008).

[32] W. M. Zheng M. P. Halsall, P. Harrison, J.-P. R. Wells, and I. V. Bradley, and M. J. Steer, Effect of Quantum-Well Confinement on Acceptor State Lifetime in $\delta$-Doped GaAs/AlAs Multiple Quantum Wells, Appl. Phys. Lett. 83, 3719 (2003). 